\title{
UNDERSTANDING THE MG II AND H $\alpha$ SPECTRA IN A HIGHLY DYNAMICAL SOLAR PROMINENCE
}

\author{
P. Heinzel ${ }^{1}$, B. SChMieder ${ }^{2}$, N. MEIN ${ }^{2}$, AND S. GunáR 3,4 \\ ${ }^{1}$ Astronomical Institute, The Czech Academy of Sciences, 25165 Ondřejov, Czech Republic; pheinzel@asu.cas.cz \\ ${ }^{2}$ Observatoire de Paris, LESIA, UMR 8109 (CNRS), F-92195 Meudon, France \\ ${ }^{3}$ School of Mathematics and Statistics, University of St Andrews, North Haugh, St Andrews KY 16 9SS, UK \\ Received 2014 November 13; accepted 2015 January 8; published 2015 February 11
}

\begin{abstract}
$\mathrm{Mg}$ II $h$ and $k$ and $\mathrm{H} \alpha$ spectra in a dynamical prominence have been obtained along the slit of the Interface Region Imaging Spectrograph (IRIS) and with the Meudon Multi-channel Subtractive Double Pass spectrograph on 2013 September 24, respectively. Single Mg II line profiles are not much reversed, while at some positions along the IRIS slit the profiles show several discrete peaks that are Doppler-shifted. The intensity of these peaks is generally decreasing with their increasing Doppler shift. We interpret this unusual behavior as being due to the Doppler dimming effect. We discuss the possibility to interpret the unreversed single profiles by using a two-dimensional (2D) model of the entire prominence body with specific radiative boundary conditions. We have performed new 2D isothermal-isobaric modeling of both $\mathrm{H} \alpha$ and $\mathrm{Mg}$ II lines and show the ability of such models to account for the line profile variations as observed. However, the $\mathrm{Mg}$ II line-center intensities require the model with a temperature increase toward the prominence boundary. We show that even simple one-dimensional (1D) models with a prominence-to-corona transition region (PCTR) fit the observed $\mathrm{Mg}$ II and $\mathrm{H} \alpha$ lines quite well, while the isothermal-isobaric models (1D or 2D) are inconsistent with simultaneous observations in the $\mathrm{Mg}$ II $h$ and $k$ and $\mathrm{H} \alpha$ lines, meaning that the $\mathrm{H} \alpha$ line provides a strong additional constraint on the modeling. IRIS far-UV detection of the $\mathrm{C}$ II lines in this prominence seems to provide a direct constraint on the PCTR part of the model.
\end{abstract}

Key words: line: profiles - radiative transfer - Sun: filaments, prominences - techniques: spectroscopic

\section{INTRODUCTION}

New instruments with advanced capabilities may bring unexpected data that represent a challenge for theoretical interpretations. This is also the case of recent prominence observations with the high-resolution Interface Region Imaging Spectrograph (IRIS; De Pontieu et al. 2014) which provides unprecedented spectral data on the $\mathrm{Mg}$ II $h$ and $k$ lines. In these lines, the prominences appear differently compared to other lines like $\mathrm{H} \alpha$ or $\mathrm{Ca}$ II and this seems to be related to their relatively high opacities. A summary of IRIS observations of a dynamic prominence, obtained in a coordinated campaign with other instruments, was recently presented by Schmieder et al. (2014). Spectral line profiles of the Mg II lines detected in this particular prominence have a complex nature never seen before and even the most simple ones present a challenge for theoretical modeling. For a review of previous work on prominence Mg II lines, see Heinzel et al. (2014) and Schmieder et al. (2014).

In this Letter, we present a first attempt to analyze the IRIS $\mathrm{Mg}$ II prominence observations in terms of the advanced nonlocal thermodynamic equilibrium (non-LTE) simulations which incorporate both fine-structure dynamics, as well as spatial variations of the kinetic temperature (PCTR-Prominence Corona Transition Region). Moreover, we use the great advantage of having simultaneous spectra of the hydrogen $\mathrm{H} \alpha$ line taken by the Multi-channel Subtractive Double Pass (MSDP) spectrograph of the Meudon solar tower. This line constrains the conditions in central parts of the prominence (Gouttebroze et al. 1993). We start our analysis using the results of one-dimensional (1D) prominence modeling of the $\mathrm{Mg}$ II lines (Heinzel et al. 2014) and extend it to

\footnotetext{
${ }^{4}$ On leave from Astronomical Institute, The Czech Academy of Sciences, Czech Republic.
}

two-dimensional (2D) in a similar manner as done by Paletou et al. (1993). Then we discuss the importance of the PCTR for the formation of optically thick $\mathrm{Mg}$ II lines in prominences. Finally, we attempt to simulate the complex $\mathrm{Mg}$ II profiles discovered by IRIS in highly dynamic parts of this prominence.

\section{PROMINENCE OBSERVATIONS}

Spectral observations of a dynamic prominence using the IRIS UV spectrograph and other instruments have been performed on 2013 September 24. These observations are described in detail in Schmieder et al. (2014). Note that in this Letter we use the intensity units for spectral lines referred to as cgs, namely, the specific line intensity in $\mathrm{erg} \mathrm{s}^{-1} \mathrm{~cm}^{-2} \mathrm{sr}^{-1} \mathrm{~Hz}^{-1}$ and the integrated line intensity in $\operatorname{erg~s} \mathrm{s}^{-1} \mathrm{~cm}^{-2} \mathrm{sr}^{-1}$.

\subsection{IRIS Spectra}

The NUV spectra (the near-UV channel of IRIS) of Mg II $h$ and $k$ lines at several slit positions and for various times were acquired. They cover the evolution and fine-structure dynamics of the observed prominence. All Mg II line profiles correspond to the Level 2 data and have been radiometrically calibrated to specific intensities using the pre-flight IRIS calibration. Two distinct types of the $\mathrm{Mg}$ II line profiles were detected: (1) single emission profiles with a marginal reversal (e.g., see profiles at pixels 200, 260, or 300 at 12:22:23 UT in Figure 1, top panels), and (2) composite emission profiles that exhibit several peaks having different intensities and being Doppler-shifted (Figure 1, bottom panels). Schmieder et al. (2014) suggested that these composite profiles are due to an overlap of several single profiles that are Doppler-shifted. This may reflect the situation when we see several moving structures (blobs) along the line of sight (LOS). An example is the pixel 150 at 12:28:20 UT. 
12:22:23 pixel 200

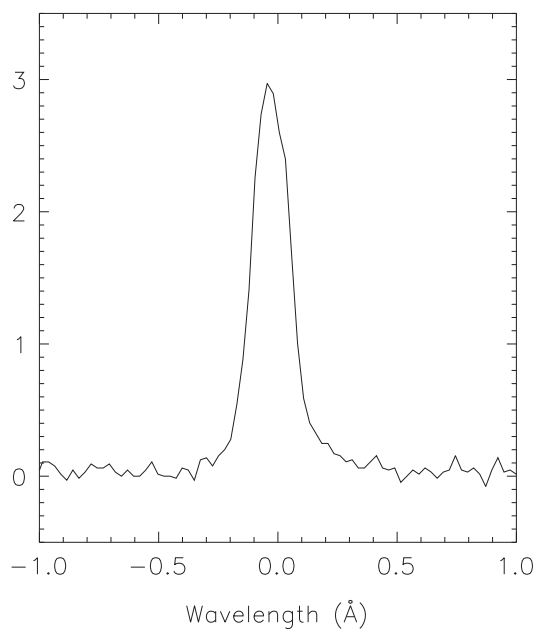

12:28:20 pixel 140

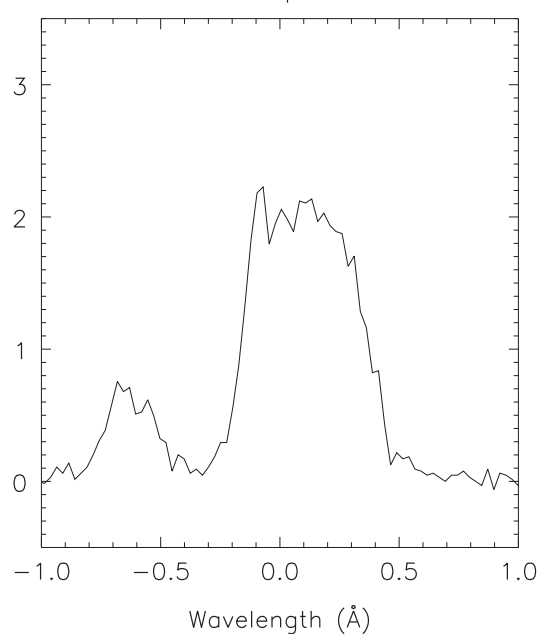

$12: 22: 23$ pixel 260

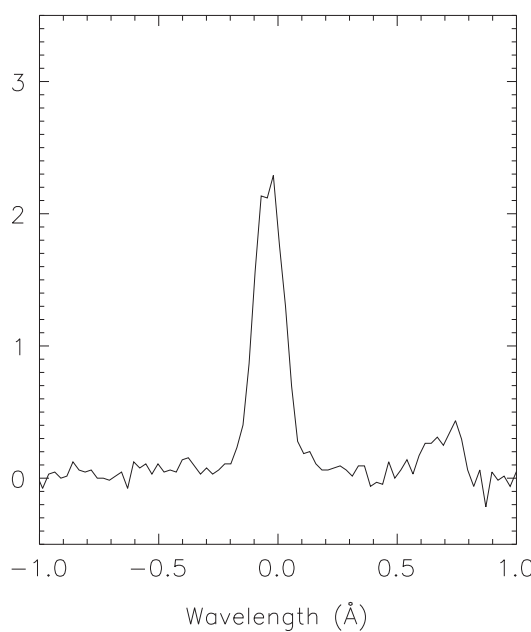

12:28:20 pixel 150

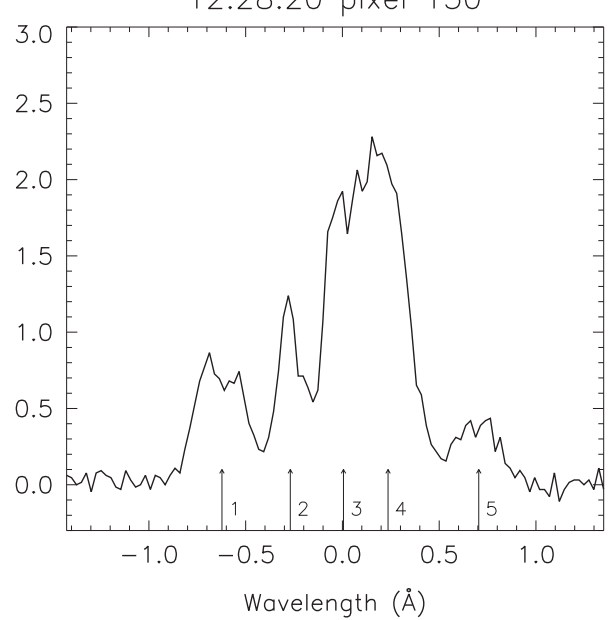

$12: 22: 23$ pixel 300

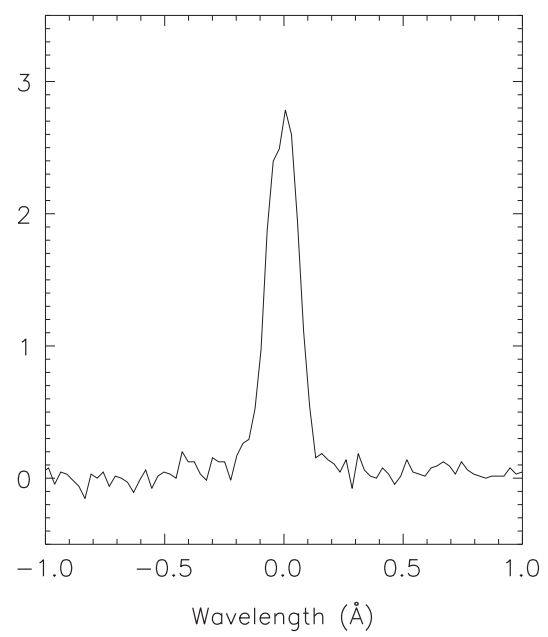

12:28:20 pixel 160

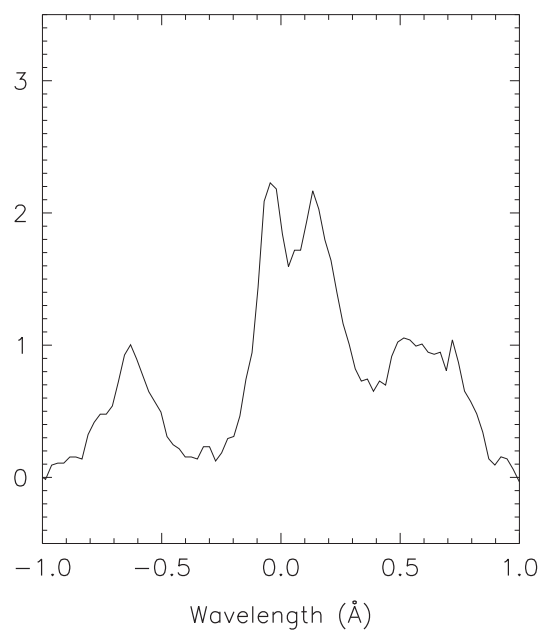

Figure 1. Single profiles of $\mathrm{Mg}$ II line (2796 $\AA$ ) obtained by IRIS—examples at 12:22:23 UT on 2013 September 24 (top panels); composite profiles of Mg II line $(2796 \AA)$ obtained by IRIS — examples at 12:28:20 UT on 2013 September 24 (bottom panels). The intensities are in units of $10^{-7} \mathrm{cgs}$.

The blobs are running along structures having a given angle with the plane of the sky (around $45^{\circ}$ ) such that the radial (vertical) velocity components are similar to the LOS ones derived from Doppler shifts. The real velocity of these blobs may reach $100 \mathrm{~km} \mathrm{~s}^{-1}$.

\subsection{MSDP at the Meudon Solar Tower}

The hydrogen $\mathrm{H} \alpha$ line was detected by the MSDP instrument mounted to the Meudon solar tower telescope; see Schmieder et al. (2014). The capability of the MSDP spectrograph is demonstrated in Mein (2002) and the MSDP observational technique is presented in Schmieder et al. (2010). The data has been reduced and radiometrically calibrated as in Gunár et al. (2012). MSDP line profiles have a Gaussian shape with integrated intensities around $10^{5} \mathrm{cgs}$, suggesting an optically thin medium in $\mathrm{H} \alpha$ (Heinzel et al. 1994). At time 12:22:14 UT (close to the observation time of IRIS) they show very small Doppler shifts up to $3-4 \mathrm{~km} \mathrm{~s}^{-1}$ which are derived from the line asymmetry. These small Doppler velocities contrast with large LOS flows detected in the Mg II lines, but one has to keep in mind that the MSDP spectral range is limited to $\pm 1 \AA$, which corresponds to $\pm 0.43 \AA$ at the wavelength position of the Mg II lines. In practice, the MSDP allows to detect Doppler-shifted profiles up to about $\pm 30 \mathrm{~km} \mathrm{~s}^{-1}$ (Figure 2).

\section{ANALYSIS OF SINGLE EMISSION PROFILES}

\subsection{Isothermal-Isobaric 1D Models}

We start with an analysis of single profiles and as a reference profile we take the profile at pixel 260 (12:22:23 UT) in Figure 1. By comparing this profile with synthetic profiles computed by Heinzel et al. (2014) for a set of 1D isothermalisobaric slab models, we immediately see that model profiles without any reversals (or only very slightly reversed) correspond to low pressures of the order of $10^{-2}$ dyne $\mathrm{cm}^{-2}$. This is also evident from Table 2 of Heinzel et al. (2014). We constructed a more extended grid of such low-pressure models that shows that the unreversed profiles have too low a central intensity as compared with the observed values $2-3 \times 10^{-7} \mathrm{cgs}$. The corresponding integrated $\mathrm{H} \alpha$ intensities are also too low compared with the MSDP data. Certain improvement is achieved by convolving the synthetic $\mathrm{Mg}$ II profiles with the IRIS instrumental profile, taken as Gaussian with the FWHM equal to the spectral resolution of the NUV channel $0.052 \AA$ (J. P. Wuelser 2015, private communication). By increasing the 

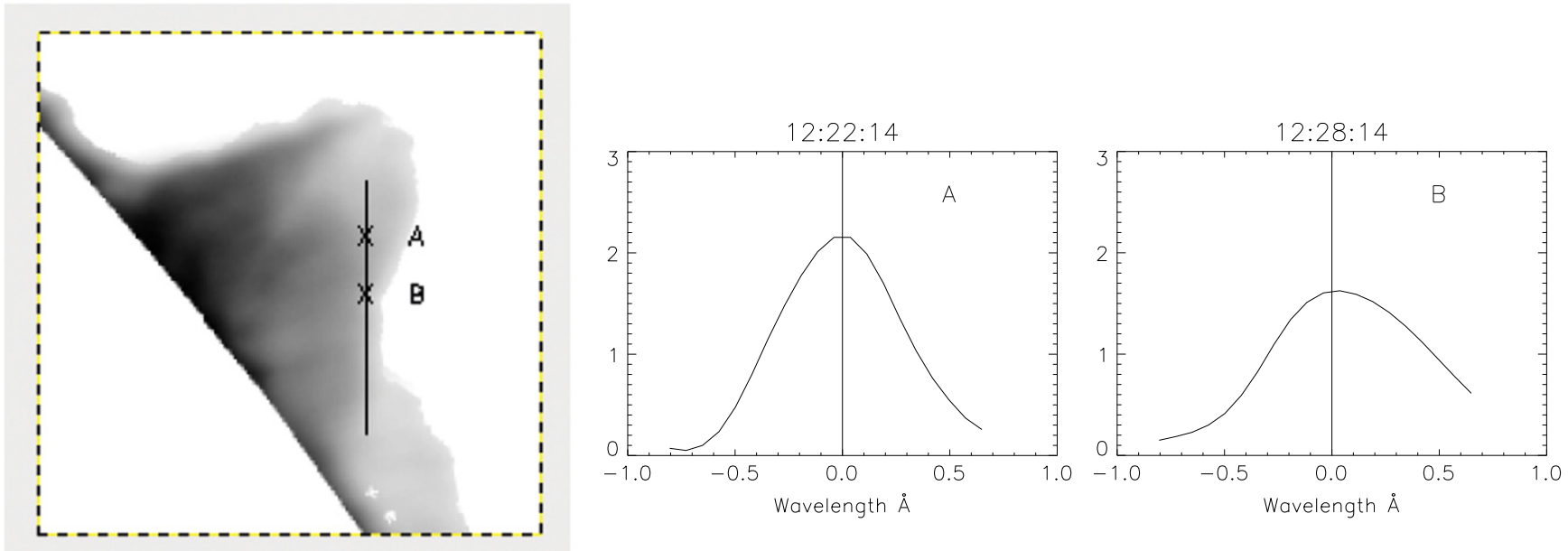

Figure 2. H $\alpha$ MSDP image of the prominence (left panel). The vertical line indicates the position of the IRIS slit and two points A and B refer to positions where H $\alpha$ profiles A and B were recorded. The representative $\mathrm{H} \alpha$ profile A is obtained at 12:22:14 UT and corresponds to the single Mg II profiles presented in the top panels of Figure 1 and profile B is obtained at 12:28:14 UT and corresponds to the composite $\mathrm{Mg}$ II profiles presented in the bottom panels of Figure 1. The intensity is in units of $10^{-6} \mathrm{cgs}$. The Doppler shift of profile A is $0.7 \mathrm{~km} \mathrm{~s}^{-1}$ and the Doppler shift of profile B is $3.3 \mathrm{~km} \mathrm{~s}^{-1}$. The line integrated intensity is $1.1 \times 10^{5}$ and $1.0 \times 10^{5} \mathrm{cgs}$, respectively, for $\mathrm{A}$ and $\mathrm{B}$.
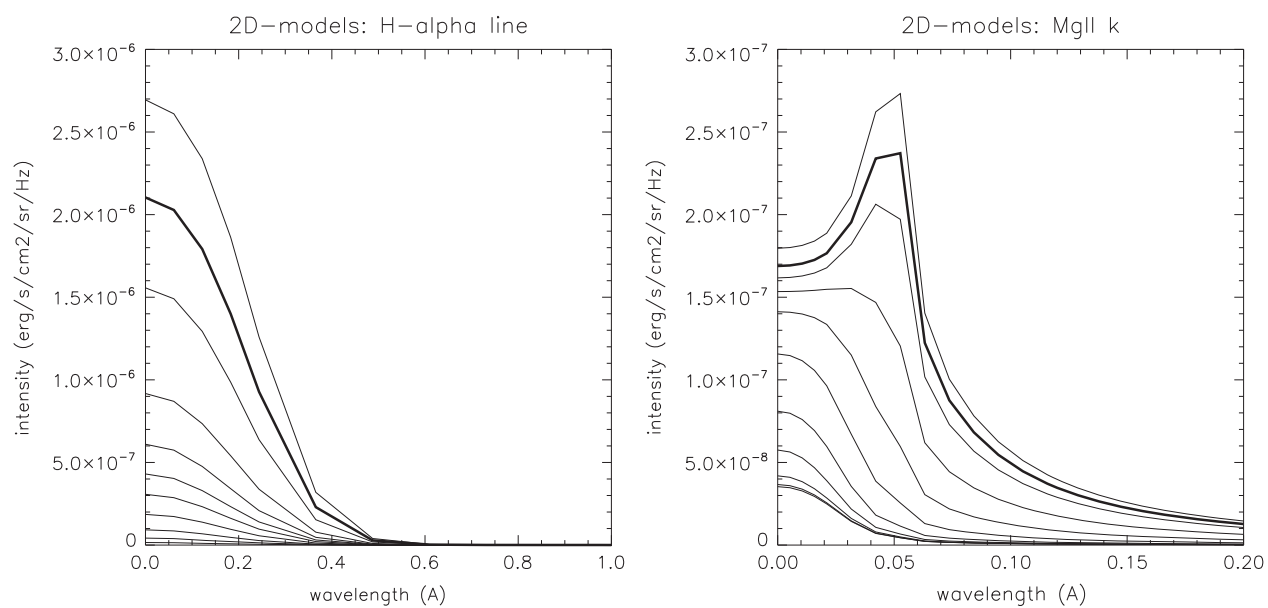

Figure 3. Height dependence of the $\mathrm{H} \alpha$ (left) and $\mathrm{Mg}$ II $k$ (right) line profiles computed with our 2D code. We show the variations of profiles from the middle heights (brightest, reversed in $k$ line) to the upper boundary. Thick lines represent an example of a reasonable fit to the IRIS and MSDP observations (Figures 1 and 2, respectively), although the $k$ line profile is reversed too much.

gas pressure and/or the geometrical extension of 1D models, the $\mathrm{H} \alpha$ intensity increases toward the observed values and also the $\mathrm{Mg}$ II line-center is enhanced. However, the Mg II line profiles are significantly reversed which is incompatible with the IRIS observations. We thus conclude that $1 \mathrm{D}$ isothermalisobaric models are not capable of explaining the unreversed profiles of the type shown in Figure 1 (upper row) consistently with the $\mathrm{H} \alpha$ intensities.

\section{2. $2 \mathrm{D}$ Models of the Prominence Body}

As a next step, we have considered 2D isothermal-isobaric models of the entire prominence body in order to see whether the spatial variations of the $\mathrm{Mg}$ II and $\mathrm{H} \alpha$ line shapes and intensities can account for their observed behavior. Such 2D models have been constructed by Paletou et al. (1993), who demonstrated rather significant changes of the $\mathrm{Mg}$ II line profiles near the top part of the 2D model, where the profiles have lower intensities and start to be non-reversed. This is a direct consequence of the 2D illumination. Very interestingly, we notice that although the $\mathrm{H} \alpha$ line integrated intensity varies with height only by about factor of 2 (Paletou 1996), the Mg II line shape and integrated intensity vary considerably, in particular at the top part of the structure. This can be easily understood because the $\mathrm{H} \alpha$ line has a relatively small optical thickness in the considered slab, while the $\mathrm{Mg}$ II lines are substantially thicker. Therefore, the 2D effects on their source function are much more important, and namely close to the surface which is not illuminated from the corona. This finding motivated us to perform new 2D modeling, using the code described in Heinzel \& Anzer (2001) and modified for Mg II using the atomic data from Heinzel et al. (2014).

The prominence body is approximated by a $2 \mathrm{D}$ rectangular box placed above the solar surface and having a vertical extension of $30,000 \mathrm{~km}$ and width $15,000 \mathrm{~km}$. Note that the infinite direction is parallel to the solar surface. The whole plasma slab is isothermal and isobaric in this simulation. We assume a full irradiation at the bottom surface of the 2D horizontal box and zero one at the top surface. Both vertical surfaces are illuminated by diluted radiation from the solar disk 
and, contrary to Paletou et al. (1993), we consider a heightdependent dilution factor.

In Figure 3 we present the results of our spectral line synthesis for model having the kinetic temperature $T=6000 \mathrm{~K}$, gas pressure $p=0.07$ dyne $\mathrm{cm}^{-2}$, and microturbulent velocity $v_{\mathrm{t}}=5 \mathrm{~km} \mathrm{~s}^{-1}$. We see the height variations of the $\mathrm{H} \alpha$ and $\mathrm{Mg}$ II $k$ line profiles, which cover the heights from central parts of the filament up to its top part. The $\mathrm{Mg}$ II $k$ line shows a similar qualitative behavior as found by Paletou et al. (1993), i.e., a decrease of the line intensity and line reversal toward the upper surface. A large variety of profiles of the $\mathrm{Mg}$ II and $\mathrm{H} \alpha$ lines suggests a possibility to improve the diagnostics using the $2 \mathrm{D}$ modeling instead of 1D. This is generally true and certainly depends on the actual shape and illumination of the entire structure. In our specific case we selected the height where the profiles of $\mathrm{Mg}$ II $\mathrm{k}$ and $\mathrm{H} \alpha$ fit the observations reasonably; see the thick profiles in Figure 3. The central reversal of the $k$ line is still non-negligible and thus this particular set of line profiles gives only slight improvement over 1D models. However, we see significant spatial variations of the line profiles in $2 \mathrm{D}$ on spatial scales of about 1 arcsec (our grid resolution at such height). This suggests that owing to uncertainties in the coalignment of the MSDP and IRIS instruments and also the calibration issues, we can also compare profiles which come from different spatial positions within the coalignment uncertainty. As mentioned above, the real structure and its illumination can be very complex and this gives even larger degree of freedom for fitting both lines.

\subsection{Back to 1D Models: Effect of PCTR on Mg II Lines}

We have seen that the expected effects of the 2D transfer are important, mainly because of a more realistic $2 \mathrm{D}$ distribution of the incident radiation. However, 2D isothermal-isobaric models are still not capable to account for the observed $\mathrm{Mg}$ II line-center intensities between 2 and $3 \times 10^{-7} \mathrm{cgs}$, while keeping the line reversal at the observed level (after convolving the synthetic profiles with the instrumental one). Because the Mg II lines are mostly optically thick, we should not neglect the potential influence of a PCTR on their formation (see Heinzel et al. 2014). Since this is completely unexplored area, we start here again with the simplified 1D models and try to see how the presence of the PCTR will affect the $\mathrm{Mg}$ II synthetic profiles. For this we use the same approach as in Heinzel et al. (2014), i.e., we parametrize the PCTR temperature structure by three quantities that are the slab-center temperature $T_{\mathrm{c}}$, boundary temperature $T_{\mathrm{b}}$, and the parameter $\gamma_{\text {tr }}$ characterizing the temperature gradient

$$
T(x)=T_{\mathrm{c}}+\left(T_{\mathrm{b}}-T_{\mathrm{c}}\right)\left(1-\frac{2 x}{D}\right)^{2 \gamma_{\mathrm{tr}}}
$$

Here $D$ is the geometrical thickness of the 1D slab and $x$ is the coordinate across the slab. Using this form of the temperature distribution, we have tried to fit the range of observed profiles of the $\mathrm{Mg}$ II $k$ line, with the central intensities between 2 and 3 $\times 10^{-7} \mathrm{cgs}$. This range approximately covers the spatial resolution of the MSDP, which is 5-10 times lower than that of IRIS. We have performed a series of trial-and-error NLTE simulations and convolved each synthetic profile with the IRIS instrumental profile. At the end we obtained almost excellent agreement with a mean observed profile, provided that we use rather low microturbulent velocity of only $2 \mathrm{~km} \mathrm{~s}^{-1}$. The other

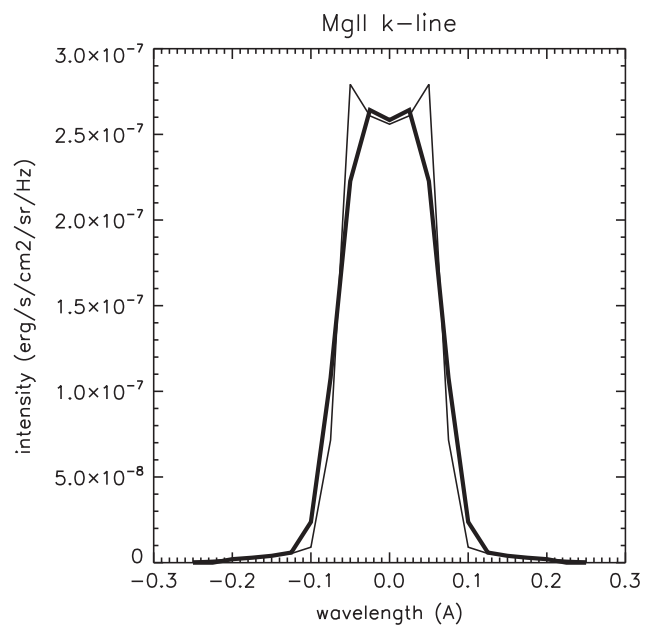

Figure 4. Synthetic $\mathrm{Mg}$ II $\mathrm{k}$ line profile computed as the best fit to mean profiles shown in Figure 1, top panel. In this case, a PCTR model was used. The thin line is the computed profile and the thick one was convolved with the IRIS instrumental profile.

parameters are $T_{\mathrm{c}}=6000 \mathrm{~K}, T_{\mathrm{tr}}=30,000 \mathrm{~K}, \gamma_{\mathrm{tr}}=5$, and the gas pressure $p=0.06$ dyne $\mathrm{cm}^{-2}$. The dilution factor for the incident radiation was evaluated at the altitude of $20,000 \mathrm{~km}$. The 1D slab thickness $D$ was taken to be only $3000 \mathrm{~km}$ but this may reflect the high degree of the prominence porosity as clearly seen from the high-resolution Hinode/Solar Optical Telescope and IRIS images (Schmieder et al. 2014). The resulting synthetic profile is shown in Figure 4 and is quite comparable with the observed profiles from Figure 1. Moreover, this 1D simulation is also consistent with the observed $\mathrm{H} \alpha$ integrated intensity of about $10^{5} \mathrm{cgs}$ which leads to the line-center optical thickness of two, while the $k$ line thickness is much larger, around 340.

\section{ANALYSIS OF COMPOSITE EMISSION PROFILES}

Composite $\mathrm{Mg}$ II profiles in this dynamic prominence exhibit several peaks. One can observe an interesting behavior that the peak intensity systematically decreases with an increasing Doppler shift. The only exception is in the vicinity of a "static" profile, where we can see a slight brightening; see Figure 1, peak No. 4. By inspection of the models presented by Heinzel et al. (2014), we see that the Mg II $k$ line-center intensity is, in most cases, saturated to values around $2 \times 10^{-7} \mathrm{cgs}$, while some peaks of the observed composite profile have their intensity as low as $10^{-7} \mathrm{cgs}$ or even lower. We suggest interpreting these systematic intensity variations in terms of the so-called Doppler dimming effect (DDE) or, in a few cases, by Doppler brightening effect (DBE; Heinzel \& Rompolt 1987). The case of $\mathrm{Mg}$ II resonance lines was studied by Heinzel et al. (2014), who demonstrated the importance of the DDE for radial velocities larger than about $20 \mathrm{~km} \mathrm{~s}^{-1}$, while below this limit the $h$ and $k$ lines are slightly brightened. The DDE at low velocities is caused by the deep reversal of the incident solar radiation. The entire composite profile is not observed in the $\mathrm{H} \alpha$ line because of a limited wavelength range of the MSDP instrument covering only $1 \AA$ from the line-center. Moreover, the individual Doppler-shifted peaks are smeared in $\mathrm{H} \alpha$ because of larger thermal broadening as compared to $\mathrm{Mg}$ II lines. In Figure 2 (profile B) we can see certain $\mathrm{H} \alpha$ line asymmetry indicating the presence of side peaks. 


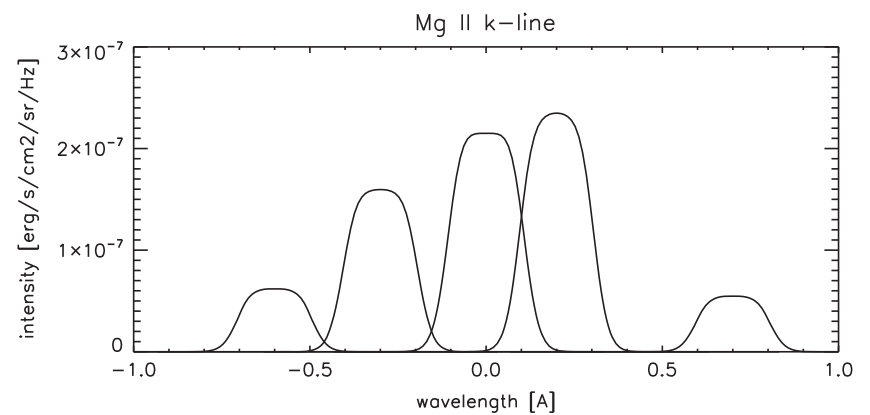

Figure 5. Simulated composite profile as described in Section 4.1. Note a qualitative similarity with the observed composite profile shown in Figure 1, pixel 150 at lower panel.

\subsection{Simulated Composite Profiles}

We attempt to simulate the shape of the observed composite profile in the following way. For each individual intensity peak we assume an identical isothermal-isobaric model which produces an unreversed profile. Then we derive the LOS velocities from the Doppler shift of individual peaks and assume that the velocity vectors have the same radial components; see Section 2.1 and Schmieder et al. (2014). For peaks numbered $1-5$, we get Doppler velocities 64, 30, 0, -20, $-75 \mathrm{~km} \mathrm{~s}^{-1}$, respectively. Finally, for each peak we compute the $1 \mathrm{D}$ model of a moving structure, using the approach of Heinzel et al. (2014). The resulting synthetic profile is shown in Figure 5, where the individual components were simply Doppler-shifted. Here we assumed that the Doppler-shifted profiles are not affected by the wing opacity of other profiles which represents certain simplification. The composite profile in Figure 5 resembles the observed one from Figure 1 and thus we believe that the surprising behavior of the latter can be due to the DDE/DBE. However, the $\mathrm{H} \alpha$ profile $\mathrm{B}$ as shown in Figure 2 represents a mean over several IRIS pixels (due to much lower MSDP spatial resolution) and thus it is difficult to interpret it in terms of composite profiles. Note also that the $\mathrm{H} \alpha$ line will be affected only by the DBE. A more quantitative explanation of such composite profiles should take into account a complex threedimensional (3D) topology of the prominence fine structure, where individual blobs have different physical conditions, may obscure each other along the LOS and will be subject to 3D velocity-dependent radiative boundary conditions. In Section 3.2 we have demonstrated how the line intensities are sensitive to even static 2D boundary conditions.

Finally, some observed Mg II line "reversals" might be just due to a composition of single-peak profiles Doppler-shifted. This seems to be the case at pixel 160 (12:28:20 UT), where we see apparently reversed profile, but its blue peak is centered at zero wavelength position, while the red one is clearly Doppler-shifted. Also, FWHM $0.4-0.5 \AA$ of such profiles can hardly be understood unless we accept very large microturbulent velocities.

\section{CONCLUSIONS AND FUTURE PROSPECTS}

In this Letter, we have attempted to understand the surprising behavior of the $\mathrm{Mg}$ II lines detected in a highly dynamic prominence. Although this prominence was classified as "quiescent," it exhibits relatively large flows visible in the optically thick Mg II lines but hardly detectable in the hydrogen $\mathrm{H} \alpha$ line. The behavior of single profiles was successfully explained by introducing a global PCTR into 1D slab models, but future, more realistic $2 \mathrm{D}$ modeling with PCTR is unavoidable. The reliability of the PCTR model used in our simulations can be tested against the observed PCTR lines. In the far-UV (FUV) channel of IRIS we can use two C II emission lines at 1334.5 and $1335.7 \AA$ (the second one is a blend of two $\mathrm{C}$ II lines). Using our optimal PCTR model, the synthetic C II line intensities were computed with the CHIANTI software (Dere et al. 1997). The agreement with the FUV intensities is very promising, taking into account the uncertainties of the FUV radiometric calibration. However, CHIANTI assumes the collisional ionization and excitation of carbon and optically thin transitions. It is therefore a challenge to compare calibrated $\mathrm{C}$ II lines from IRIS with consistent NLTE modeling of carbon lines. The formation temperature of the C II lines was estimated in De Pontieu et al. (2014) to be around $2 \times 10^{4} \mathrm{~K}$, quite nicely in the range of the PCTR temperatures considered in our model. The next step in modeling of the PCTR will be an implementation of the 2D code for $\mathrm{Mg}$ II lines as described here to multithread structures discussed in Gunár (2014).

In the second part of our study, we have demonstrated the importance of the DDE/DBE on the $\mathrm{Mg}$ II line emission considering velocities consistent with the observed Doppler shifts. Namely, the dimming effect can easily account for very low line-center intensities below $10^{-7} \mathrm{cgs}$ which have been detected in this dynamic prominence. Several structures (blobs) moving along the LOS produce Doppler-shifted profiles which are well resolved because of their narrowness. For radial velocities larger than about $20 \mathrm{~km} \mathrm{~s}^{-1}$ there is a clear tendency for an intensity decrease with increasing Doppler shift. As a next step, fully 2D/3D transfer models have to be constructed taking into account the velocity-dependent radiative boundary conditions.

P.H. and B.S. acknowledge the Meudon Observatory and the Ondřejov Observatory, respectively, for support and hospitality. We thank P. Schwartz for computing C II lines using the CHIANTI software and H. Tian for providing us with Figure 1. This work was partially supported by the grant 209/12/0906 of the Grant Agency of the Czech Republic and by the project RVO 67985815. S.G. acknowledges support from the European Commission via the Marie-Curie Actions-Intra European Fellowship Project No. 328138. We thank U. Anzer and P. Mein for fruitful discussions and comments. Constructive comments by the anonymous referee are also highly appreciated.

\section{REFERENCES}

De Pontieu, B., Title, A. M., Lemen, J., et al. 2014, SoPh, 289, 2733

Dere, K. P., Landi, E., Mason, H. E., Monsignori Fossi, B. C., \& Young, P. R. 1997, A\&AS, 125, 149

Gouttebroze, P., Heinzel, P., \& Vial, J. C. 1993, A\&AS, 99, 513

Gunár, S. 2014, in IAU Symp. 300, Nature of Prominences and Their Role in Space Weather, ed. B. Schmieder, J.-M. Malherbe, \& S. T. Wu (Cambridge: Cambridge Univ. Press), 59

Gunár, S., Mein, P., Schmieder, B., Heinzel, P., \& Mein, N. 2012, A\&A 543, A93

Heinzel, P., \& Anzer, U. 2001, A\&A, 375, 1082

Heinzel, P., Gouttebroze, P., \& Vial, J.-C. 1994, A\&A, 292, 656

Heinzel, P., \& Rompolt, B. 1987, SoPh, 110, 171

Heinzel, P., Vial, J.-C., \& Anzer, U. 2014, A\&A, 564, A132

Mein, P. 2002, A\&A, 381, 271

Paletou, F. 1996, A\&A, 311, 708

Paletou, F., Vial, J.-C., \& Auer, L. H. 1993, A\&A, 274, 571

Schmieder, B., Chandra, R., Berlicki, A., \& Mein, P. 2010, A\&A, 514, A68

Schmieder, B., Tian, H., Kucera, T., et al. 2014, A\&A, 569, A85 